\title{
Measurement of Differential Pressure by Optical Interferometry with Multimode Tunable Lasers
}

\author{
Marcelo T. Saita ${ }^{1}$, Eduardo A. Barbosa ${ }^{2, *}$, Francisco T. Degasperi ${ }^{2}$ and Niklaus U. Wetter ${ }^{3}$ \\ ${ }^{1}$ Instituto de Pesquisas Tecnológicas, São Paulo, Brazil \\ ${ }^{2}$ Unidade de Pós-Graduação, Extensão e Pesquisa, CEETEPS, São Paulo, Brazil \\ ${ }^{3}$ Centro de Lasers e Aplicações, Instituto de Pesquisas Energéticas e Nucleares, São Paulo, Brazil
}

Received 3 April 2020; Accepted 20 October 2020

\begin{abstract}
Determining the deformation of a capacitive membrane is a well-known pressure measurement technique. A novel technique is presented for pressure measurement based on low coherence speckle interferometry (LCSI) to optically evaluate the deformation of a membrane submitted to a pressure differential. By processing the low spatial frequency speckle fringe pattern through four-stepping and phase unwrapping methods the membrane contour was determined. A tunable multimode red diode laser is the light source. A circular aluminum membrane was submitted to pressure values up to $90 \mathrm{kPa}$ resulting in a curve of the central membrane deformation as a function of the pressure. An External Cavity Diode Laser (ECDL) was used for laser tuning thus changing the interferogram spatial frequency and the sensitivity of the measurement process. The interferometry results were compared with those obtained experimentally by a feeler clock and by an analytical expression. The possibility of applying this technique in quasi real-time testing is explored.
\end{abstract}

Keywords: Speckle interferometry, laser tuning, pressure measurement.

\section{Introduction}

Pressure is the most measured physical quantity in the world. Hence, it is expected that there are several optical techniques for pressure measurement and sensing. Indeed, over the years, the use of optical methods for pressure measurement has been proposed by several authors. Those methods are generally based either on geometrical optics or on wave optics to measure the deformation of membranes or diaphragms in the range of hundreds of micrometers up to several millimeters. In the field of geometrical optics, pressure is measured by taking advantage of polarization properties of light undergoing total internal reflection [1]; optical fibers have been extensively used in pressure sensing investigations in loop ring-down configurations [2,3], in combination with a micro Fabry-Perot interferometer [4] and in a fiber-Bragggrating scheme [5]; other works used interferometry for pressure measurement by means of a Michelson interferometer [6], moiré interferometry [7], digital holographic interferometry [8] and digital speckle interferometry $[9,10]$. In these latter works, the deformation of micro-membranes submitted to pressure differentials is measured by double-exposure holography and doubleexposure digital speckle interferometry.

In this work we propose a method which uses low coherence speckle interferometry (LCSI) for pressure measurement and sensing by determining the deformation undergone by a membrane submitted to a pressure differential. In LCSI the interferometer is illuminated by two or more collinear laser beams with slightly different

*E-mail address: ebarbosa@fatecsp.br

ISSN: $1791-2377$ @ 2020 School of Science, IHU. All rights reserved.

doi:10.25103/jestr.136.03 wavelengths, so that the resulting image of the analyzed surface appears modulated by low-frequency contour fringes. Thus, once the membrane shape for null pressure differential is known, the deformation after pressure increase is easily determined by applying phase stepping and phase unwrapping procedures. In whole-field interferometric methods, whether by holography or by speckle interferometry, different configurations were proposed for obtaining contour interferograms. In references [11,12] the interferometer was illuminated by a single diode laser emitting simultaneously several longitudinal modes and the spatial period of contour interferograms was closely related to the laser free-spectral range (FSR). Hence, since the FSR of the laser is a fixed parameter, the sensitivity of the measurements in those works could not be adjusted. In order to overcome this limitation, variable spatial period fringe patterns were obtained by using two conveniently aligned lasers tuned by drive current selection or by temperature adjusting [13,14]. A drawback of this configuration in turn is the difficulty to simultaneously lock the wavelengths of both lasers to keep the interferogram constant in time.

For fringe temporal stability purposes the interferometer was illuminated in this work by a single tunable External Cavity Diode Laser (ECDL) based on the Littman-Metcalf (LM) method, which allows selecting the most suitable emission parameters according to a required measurement sensitivity $[15,16,17]$. The LM setup used in this work has a controlled feedback with a reflective diffraction grating in order to achieve and properly control a two-wavelength emission. Since those wavelengths are obtained from the same gain line, the mutual stability is much higher than the one obtained in previous configurations, which resulted in much more stable fringe patterns. 
For each pressure differential, we measured the membrane shape, and compared the optical experimental values with the ones measured with the help of a feeler clock. Moreover, the values obtained by our technique were also compared with results obtained by an analytical expression.

\section{Deformation Measurement}

\subsection{Low coherence DSPI}

The principle of the method is obtaining a curve between the pressure differential and the membrane deformation measured by LCSI. Consider the interference of a wave scattered by a rough surface object and a reference wave, both with wavelengths $\lambda_{1}$ and $\lambda_{2}$ originating from a multimode laser onto a CCD target. If both waves have the same amplitude, the intensity of the speckle pattern is given [17,18] by equation (1),

$$
I(x, y)=I_{0} \cos ^{2}\left[\frac{\pi\left(\Gamma_{s}(x, y)-\Gamma_{R}\right)}{\lambda_{s}}\right]
$$

Where $\Gamma_{s}(x, y)$ is the optical path of the beam impinging the object on point $(x, y), \Gamma_{R}$ is the optical path of the planar wavefront reference beam and $\lambda_{s}=\lambda_{1} \lambda_{2} /\left|\lambda_{1}-\lambda_{2}\right|$ is the synthetic wavelength. Equation (1) shows that the object image appears modulated of a contour interferogram, such that each fringe corresponds to a plane of constant elevation. For normal incidence on the test object, the distance between two adjacent planes is $\Delta \mathrm{z}=\lambda_{\mathrm{s}} / 2$. By properly selecting the wavelength difference $\lambda_{1}-\lambda_{2}$, the spatial frequency of the interferogram can be properly adjusted. Phase evaluation algorithms allow determining the membrane shape when it is submitted to a pressure differential

\subsection{Analytical deformation model}

Young and Gordon described the deformation of planar surfaces with several geometries under several boundary conditions [19]. For circular plates with fixed borders submitted to a uniform pressure distribution, the maximal deformation $z_{\max }$ is given by (in SI units)

$$
\frac{p D^{4}}{E t^{4}}=K_{1} \frac{z_{\max }}{t}+K_{2}\left(\frac{z_{\max }}{t}\right)^{3}
$$

where $p$ is the uniformly distributed pressure, $t$ is the plate thickness, $D$ is the plate diameter and $E$ is the elasticity modulus of the plate material. When the borders are both fixed and held, factors $K_{1}$ and $K_{2}$ are given by

$$
K_{1}=\frac{5.33}{1-v^{2}}
$$

and

$$
K_{2}=\frac{2.6}{1-v^{2}}
$$

where $v$ is the Poisson coefficient. Equations (2) and (3) are most accurate for deformations not exceeding half of the membrane thickness.

\section{Experiments}

\subsection{ECDL for laser tuning}

The Littman-Metcalf (LM) feedback technique can be used to conveniently tune wavelengths $\lambda_{1}$ and $\lambda_{2}$ and the value of $\Delta z$, thus allows the adjustment of the measurement sensitivity according to the deformation magnitude of the membrane. The LM setup is shown in figure 1. The technique uses an external cavity to control the laser feedback by using a diffraction grating in order to select and stabilize the emission wavelength [15]. As the output laser beam impinges the grating, the $1^{\text {st }}$ order beam in turn is reflected by mirror M1 back to the grating and is re-injected into the diode laser. Due to the divergence of the diffracted waves, MI can be properly tilted in order to re-inject some specific modes which are reinforced inside the laser diode and have enough gain to oscillate. Dual-mode laser operation is obtained since both the mode given by the diode laser cavity and the mode given by the external cavity compete for the available gain [20]. Stable operation can be also more easily obtained by conveniently adjusting the laser drive current. Since only the $0^{\text {th }}$-order diffracted beam is used to illuminate the interferometer, the configuration of the LM setup as shown in figure 1 assures that tilting mirror M1 does not affect the beam alignment at the interferometer.

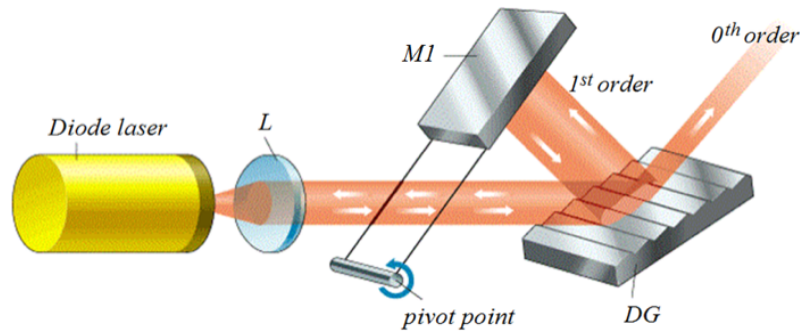

Fig. 1 Littman-Metcalf ECDL: L, lens; M1, mirror; DG, diffraction grating

\subsection{Interferometer}

The speckle interferometer is shown in Figure 2. The beam leaves the ECDL and is split into reference and object arms at beam splitter BS1. In the reference arm the beam is reflected by mirror M2, by a right-angle prism translator PT and by a mirror PZT-M, supported by a piezoelectric transducer. Before reaching the PZT-M the reference beam passes also through lens L1 and by a spatial filter SF to impinge the CCD camera. The prism PT is translated by a micrometric screw to perform four-stepping procedures [21] and the PZT-M vibrates at $5 \mathrm{~Hz}$ in order to obtain the fringe pattern by the subtraction method [18]. In the object arm the beam diameter is collimated and enlarged by the beam expander BE to illuminate the membrane with normal incidence with the help of beam splitter BS2. Beam splitter BS3 partially deviates the reference beam for spectrum monitoring at the spectrum analyzer SA. The object beam is reflected by mirror M3 and is coupled to the reference beam by beam splitter BS4. Lens L3 is the imaging lens for the object and also allows that the reference beam hits the CCD target with a planar wavefront.

The software used in this work for data acquisition and image processing (LABVIEW®) can be used to successively acquire two frames and perform their subtraction in order to obtain a single background-free speckle pattern. Between 10 and 20 of such patterns are averaged to get the contour speckle interferogram, i.e., a high spatial frequency speckle pattern modulated by low spatial frequency contour fringes. By 
applying Fast Fourier Transform (FFT) and low-pass spatial filtering, a smooth contour pattern was finally obtained. The whole process described above takes approximately 2 s. For the sake of comparison, the membrane deformations were measured by a feeler clock (Mitutoyo, series 513, precision $0.01 \mathrm{~mm}$ ) also.

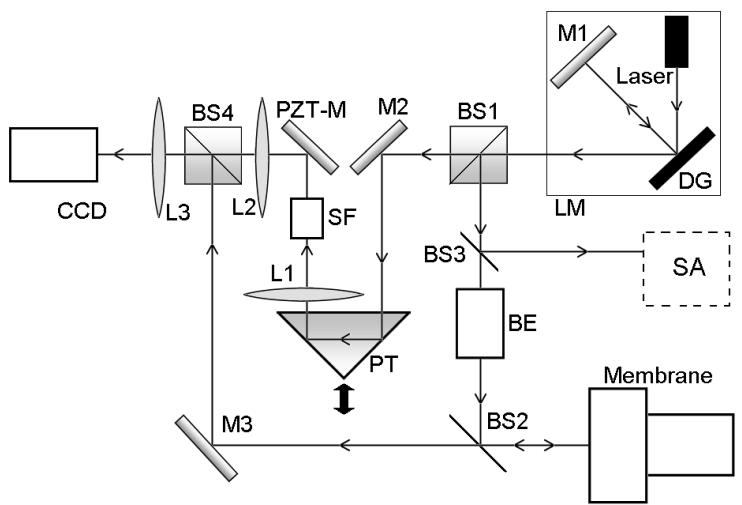

Fig. 2 Optical setup of the speckle interferometer: M1 to M3, mirrors; L1 to L3, lenses; BS1 to BS4, beam splitters; BE, beam expander; PZT$\mathrm{M}$, piezoelectric transducer-supported mirror; $\mathrm{PT}$, prism translator; SF, spatial filter; DG, reflective diffraction grating; SA, spectrum analyzer; CCD, camera.

\subsection{Vacuum system}

The aluminum membrane assembly is shown in figure 3 . This setup measures "gauge pressure", i.e., positive pressure values are larger than the atmospheric pressure, "negative pressure" values are smaller than the local atmospheric pressure [22], adopting the way when using the gauge pressure, the local atmospheric pressure being zero). The laser impinges the left side of the membrane submitted to atmospheric pressure, while at the right side of the membrane there is air at the measured pressure.

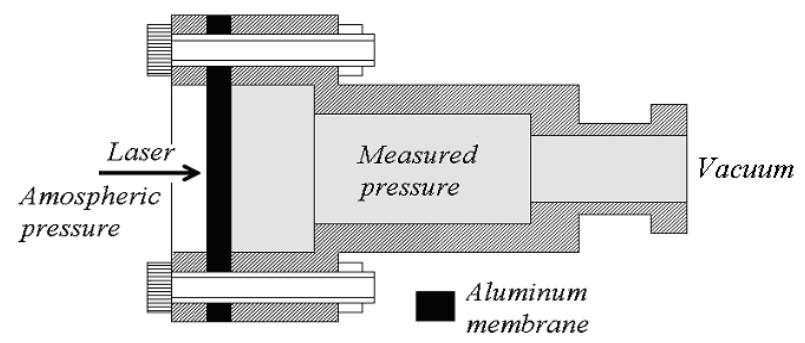

Fig. 3. Membrane assembly

The pressure measurement was performed by an indirect method in which the deformation of a diaphragm or membrane (sensor element), subjected to a pressure differential, was measured and used to calculate the pressure. Known pressure values were applied to a diaphragm with the aid of a standard vacuum sensor; a double-stage vane type vacuum pump was used to produce pressure values. For each pressure value the respective membrane deformation was measured both optically and mechanically.

Figure 4 shows the vacuum circuit used to characterize the sensor membrane. Components have been installed to make the measurement more reliable; for example, the installation of metal bellows prevents the vibration of the vacuum pump from propagating to the membrane. The vacuum pump filter prevents oil vapor from contaminating the membrane housing. The installed valves make it easy to adjust and control the desired pressure. The experimental set-up has been verified as stable and safe, responding adequately to the requirements necessary to obtain accurate measurements.

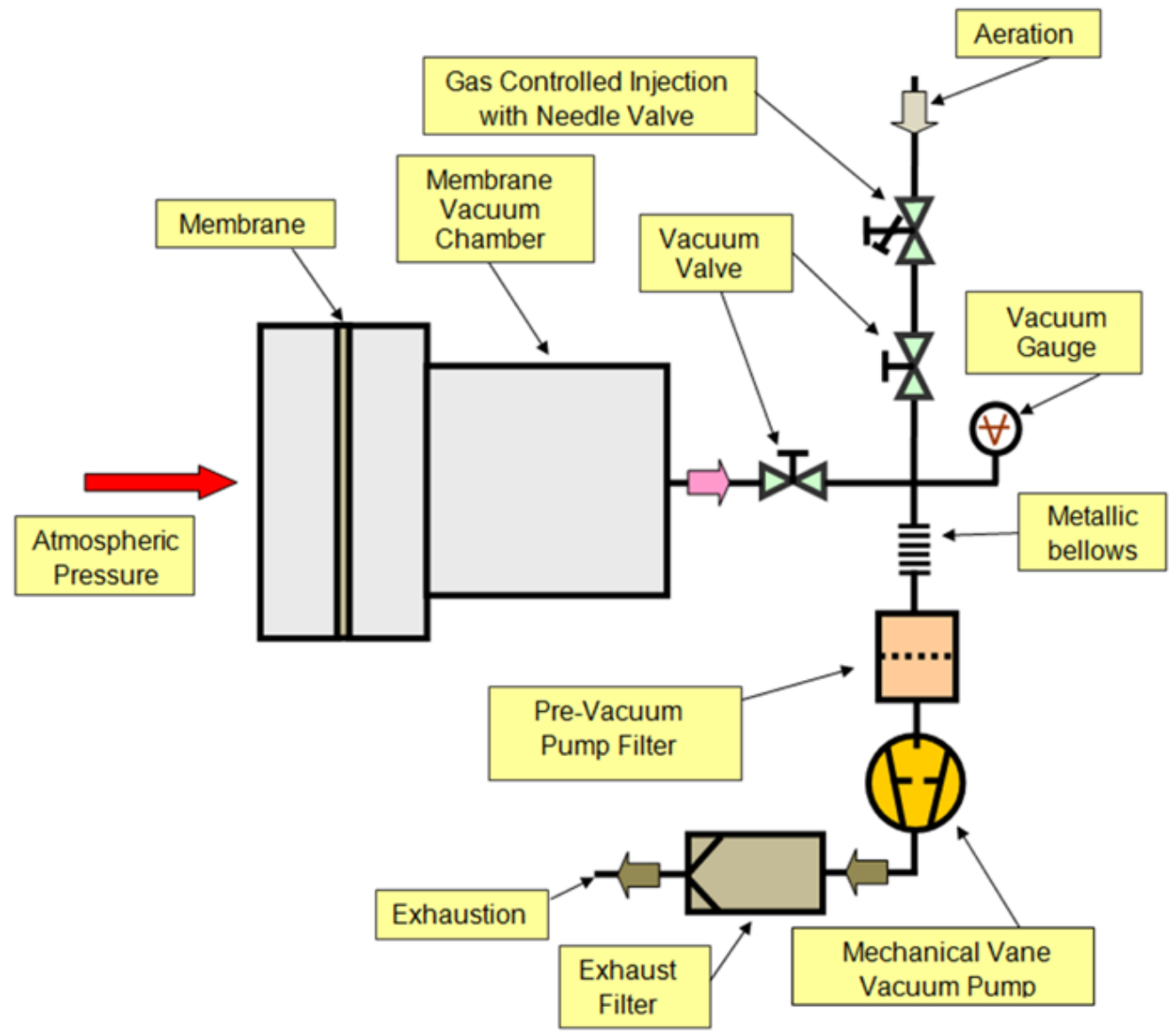

Fig. 4. Circuit diagram of the vacuum system 
The pressure differential was applied to the membrane by means of a two-stage rotary vane mechanical pump (also called mechanical oil sealed pump; LEYBOLD, model D5E) with pumping speed of $5 \mathrm{~m}^{3} / \mathrm{h}$ obtaining a minimum final pressure of $9 \times 10^{-3} \mathrm{mbar}$. The fine pressure adjustment was performed by means of needle-type valves. The vacuum gauge used (ASHCROFT, model $\mathrm{v} / 100 \mathrm{psi}$ ) operates in the range of $-100 \mathrm{kPa}$ to $700 \mathrm{kPa}$ with a resolution of $0.1 \mathrm{kPa}$.

\section{Results and Discussion}

\subsection{Phase mapping and phase unwrapping}

The four-frame procedure consists in sequentially phase shifting one of the interfering beams in the optical setup by $\pi / 2$ phase values [21]. Figure $5 \mathrm{a}$ shows the phase map resulting from a particular membrane deformation. Figure $5 \mathrm{~b}$ shows the unwrapped phase obtained by the branch-cut method [23], where the darker regions correspond to lower membrane vertical displacement levels. In summary, both figures evidence that the center of the membrane undergone maximum displacement. The deformation distribution across the membrane surface is obtained by converting the gray levels of the unwrapped phase into height coordinate values.

\subsection{Deformation measurement and calculation}

The Aluminum alloy membrane used in this work has a thickness of $423 \pm 3 \mu \mathrm{m}$ and a diameter of $55.00 \pm 0.04 \mathrm{~mm}$. The parameters used in equation (2) were $\mathrm{E}=72 \mathrm{GPa}$ for the elasticity modulus and $v=0.33$ for the Poisson coefficient, measured at the Technological Research Institute (IPT). The maximal displacements were calculated for several pressure values.

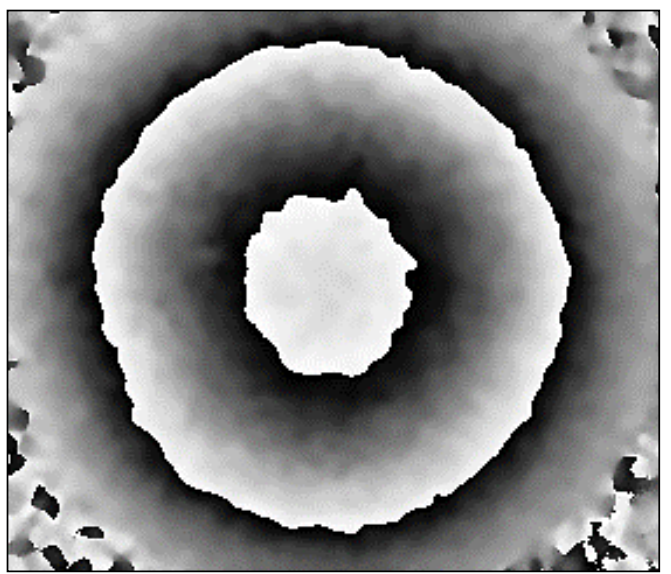

(a)

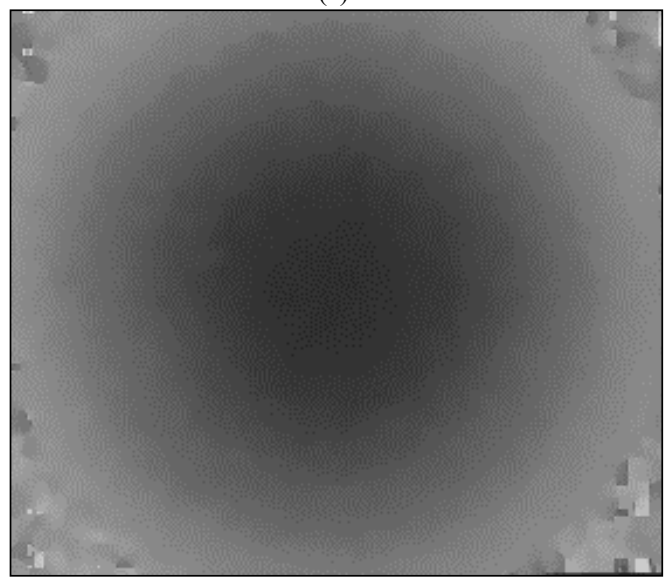

(b)

Fig. 5 a-Phase mapping; $b$ - unwrapped phase
Figure 6 displays the results calculated from equation (2) and compares them with the maximum displacement values obtained by LCSI and with the results measured by the feeler clock, showing a good agreement of the data.

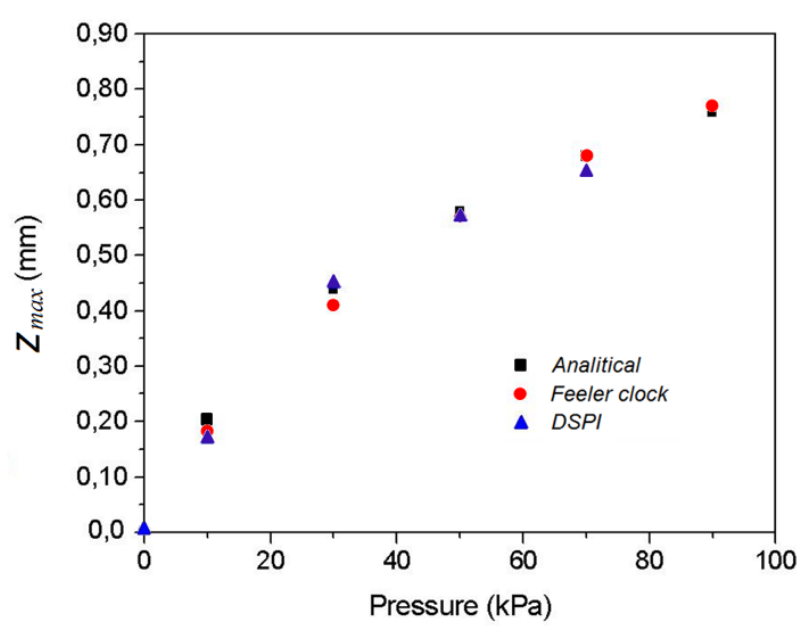

Fig. 6 Maximum deformation of the membrane submitted to the pressure differential. Triangles to speckle interferometry; circles to feeler clock and squares to analytical results.

\subsection{Nearly real-time acquisition}

In this section we consider the possibility of using low coherence DSPI for performing pressure measurements in a nearly real-time fashion. Figure $7 \mathrm{a}$ shows a real time fringe pattern $(\Delta z=0.48 \mathrm{~mm})$ of the deformed membrane submitted to a $90-\mathrm{kPa}$ pressure differential, while figure $7 \mathrm{~b}$ shows the intensity profile of those fringes taken along the line P-Q in figure $7 \mathrm{a}$. Both figures are acquired simultaneously. The $y$ coordinates of the fringe minima (see points A,B, C and D in figure $7 b$ ) are determined, and once the synthetic wavelength is known, the $z$ deformation values corresponding to the dark fringes as a function of $y$ can be plotted while the measurement is performed, as shown in figure 7c (crosses). This graph plots also the whole membrane deformation $z$ obtained with the help of the unwrapped phase (squares), similarly to the one obtained in figure $6 \mathrm{~b}$. As expected, both plots are very close, showing that determining the positions of the dark (or bright) fringes enables the pressure measurement in nearly real time. Similarly, figure $7 \mathrm{~d}$ compares the deformation at the dark fringes with the deformation obtained from the unwrapped phase. In this measurement, the distance between two adjacent planes was $\Delta z=0.44 \mathrm{~mm}$.

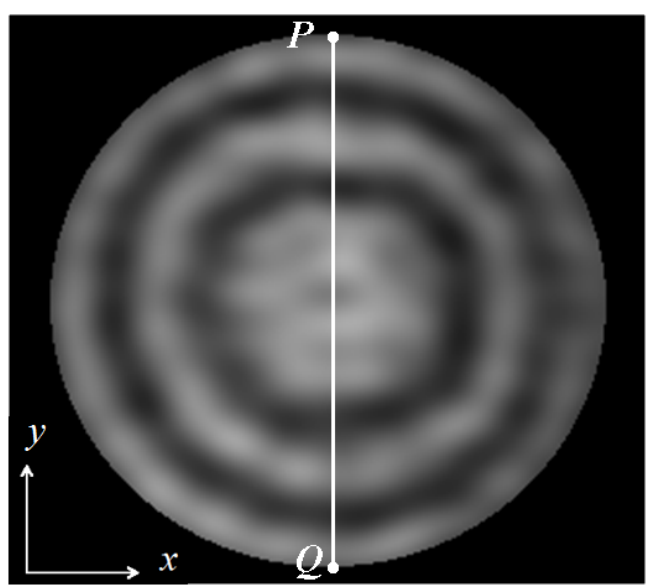

(a) 
Marcelo T. Saita, Eduardo A. Barbosa, Francisco T. Degasperi and Niklaus U. Wetter/

Journal of Engineering Science and Technology Review 13 (6) (2020) 17 - 21

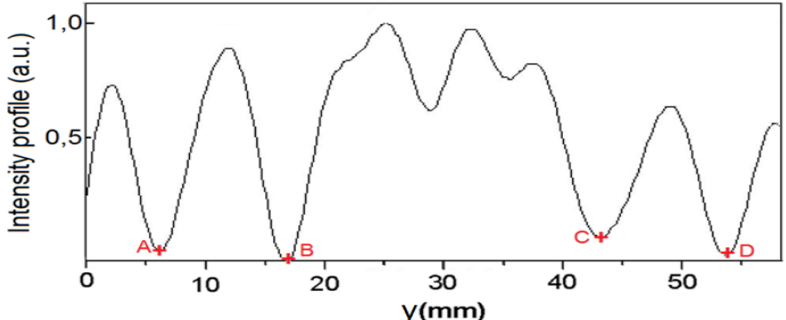

(b)

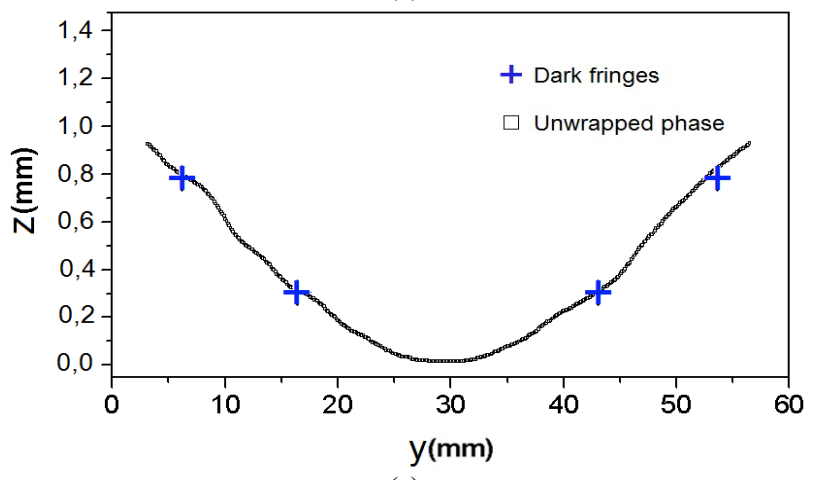

(c)

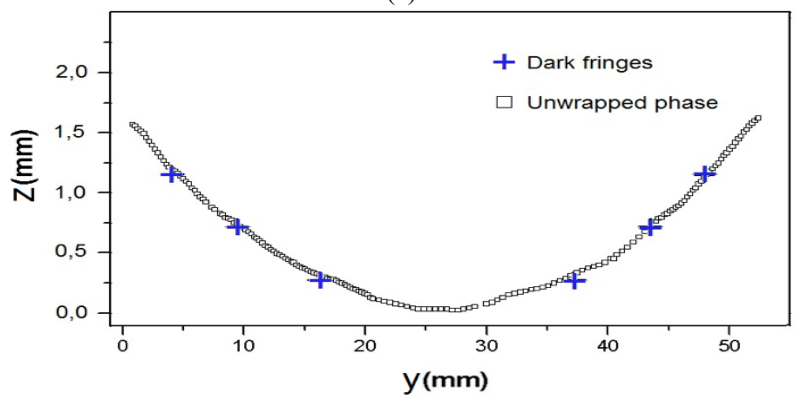

(d)

Fig. 7 a - Fringe pattern $(\Delta \mathrm{z}=0.48 \mathrm{~mm})$ of the membrane submitted to a $90-\mathrm{kPa}$ pressure differential; $\mathrm{b}$ - intensity profile of the fringe pattern along the line PQ; $\mathrm{c}$ - deformation of the membrane along line PQ taken from figure $8 \mathrm{~b}$ (crosses) and from the unwrapped phase (squares); $\mathrm{d}-$ deformation along the same line for other pressure differential and for $\Delta z$ $=0.44 \mathrm{~mm}$

\section{Conclusion}

A LCSI method for pressure measurement by membrane deformation has been successfully demonstrated. It has been shown that the experimental results were in very good agreement with the ones obtained by the feeler clock and by the analytical expression. The small discrepancies between those results can be mainly attributed to slight fluctuations in the laser emission which may cause some variations in the synthetic wavelength, despite the use of the LM setup, which in turn greatly contributed to the emission stabilization. By tuning the laser emission with the help of the LM it was possible to conveniently adjust the synthetic wavelength according to the membrane deformation, which enables working with membranes of materials with different responses. It has been pointed out that the pressure differential can be obtained both by conventional fringe evaluation methods (phase stepping and phase unwrapping) or by directly determining the fringe positions. The latter does enable pressure measurement in quasi real time, enabling much faster testing.

The results obtained in this work also point out to possible interesting applications, such as the determination of Young's modulus of materials once their dimensions and loading are known, evaluation of plate uniformity and material anisotropies, and the determination of the mechanical properties of large pieces, in the form of a plate, without their disassembly

This is an Open Access article distributed under the terms of the Creative Commons Attribution License

\section{References}

1. J. F. M. Domenegueti, S. C. Zilio, Applied Optics 53, 8 (2014).

2. Y. Jiang, J. Zhao, D. Yang, D. Tang, Opt. Comm. 283, 20 (2010).

3. D. Tang, D. Yang, Y. Jiang, J. Zhao, H. Wang, Opt. Lasers Engng 48, 12 (2018).

4. X. Zou, N. Wu, Y. Tian, C. Niezrecki, J. Chen, X. Wang, Opt. Lasers Engng 51, 2 (2013).

5. Z. Zhiguo, S. Chunyan, L. Luming, Opt. Comm. 411 (2018).

6. L. Lipiäinen, K. Kokkonen, M. Kaivola, Opt. Lasers Engng 88 (2017).

7. C. Sciammarella, P. Piroozan, Opt. Lasers Engng. 45, 5 (2007).

8. N. K. Mohan, P. K. Rastogi, Opt. Lasers Engng. 47, 2 (2009).

9. J.M. Huntley, H.O. Saldner, Opt. Las. Engng 23, 5 (1995).

10. P.K. Upputuri, M.P. Kothiyal, S. Umapathy, N.K. Mohan, Defense Science Journal 61, 5 (2011).

11. E.A. Barbosa, A.C.L. Lino, Appl. Opt. 46, 14 (2007).

12. E.A. Barbosa, S.C.dos Santos, Opt. Comm. 28, 5 (2007).
13. E.A. Barbosa, D.M. Silva, C.E. Nascimento, F.L. Galvão, J.C.R. Mittani, Opt. Las. Engng. 51, 7 (2013).

14. D. Francis, D. Masiyano, J. Hodgkinson, R.P. Tatam, Meas. Sci. Technol. 28, 5 (2015).

15. M.G. Littman, H.J. Metcalf, Appl. Opt. 17, 14 (1978).

16. K. Liu, M.G. Littman, Opt. Lett 6, 3 (1981).

17. D.M. da Silva, E.A. Barbosa, N.U. Wetter, G. Cardoso, Applied Physics B 118 (2015).

18. E. Hack, B. Frei, R. Kästle, U. Sennhauser, Appl. Opt. 37, 13 (1998).

19. W.C. Young, R.G. Budynas, Roark's formulas for stress and strain. McGraw-Hill, New York, p. 448 (2002).

20. W. E. J. Lamb, Phys. Rev. 134, A129 (1964).

21. K. Creath, Prog. Opt. 26 (1988).

22. L. Cuscó et al, Guide to the Measurement of Pressure and Vacuum, The Institute of Measurement and Control, London (1998).

23. B. Gutmann, H. Weber, Appl. Opt. 38, 2 (1999). 